\title{
ESTUDO COMPARATIVO DA AMAMENTAÇÃO EM CASAS E EM CRECHES PAULISTANAS E NO INTERIOR DO PIAUÍ ${ }^{1}$
}

\author{
BREAST-FEEDING BRAZILIAN CHILDREN: \\ A COMPARATIVE STUDY
}

Neusa Guaraciaba dos Santos Oliveira ${ }^{2}$ Elaine Pedreira Rabinovich ${ }^{3}$

SANTOS-OLIVEIRA, N. G. d.; RABINOVICH, E. R Estudo comparativo da amamentação em casas e em creche paulis^itanas e no interior do Piauí. Rev. Bras. Cresc. Desenv Hum., Sao Paulo, 9(1), 1999.

Resumo: O presente trabalho objetivou discutir questões atinentes à amamentação comparando o sistema de aleitamento em três contextos de desenvolvimento diversos: um grupo de crianças de famílias de baixa renda, de 0-24 meses de idade, moradoras de uma região urbana da cidade de São Paulo; um grupo de crianças paulistanas, de 4-12 meses, frequentadoras de uma creche no local de trabalho da mãe; um grupo de crianças de 0-3 anos, moradoras da região do Cocal do Estado do Piaui. Os dados foram obtidos por: entrevista com a mãe; observação e videofilmagem; observação e registro do ambiente; entrevista familiar. Os sistemas de aleitamento foram comparados quanto a: idade e motivo do desmame; esquema de aleitamento; e suplementação ao aleitamento; locus do aleitamento; padronizações norrnatizadoras. Concluiu-se que: a idade do desmame esteve associada à motivação materna e à presen^\$a/ausência de uma rede de apoio, em que norma s eram confrontadas com a realidade do cotidiano sócio-familiar; generalizações para a população total deveriam ser evitadas sem a consideração de variaveis contextuais; e que os valores da cultura de ocidental de individualismo, separação e autonomia parecem contraditórios com as práticas de aleitamento.

Palavras-chave: amamentação; contexto de desenvolvimento; rede de apoio; motivação materna; padronizações normatizadoras; cotidiano sécio familiar.

\section{INTRODUÇÃO}

No Brasil, pode-se colocar em discussão se as campanhas do Ministério da Saúde para promover o aleitamento materno têm ou não alcançado o objetivo almejado. Para ISLER, LEONE \& QUINTAL (1989), na cidade de São Paulo a idade média do desmame foi apenas 2,8 meses, enquanto para VENÂNCIO e
MONTEIRO (1998) teria havido uma expansão considerável da prática da amamentação no país, com um aumento proporcional mais considerável na região urbana Centro-Sul, entre mulheres de maior poder aquisitivo e maior escolaridade. Estes autores advogam a amamentação exclusiva até 4-6 meses de vida, continuando até os 2 anos complementado por outros alimentos (p. 41).

1 Trabalho apresentando no 11 Congresso Brasileiro de Psicologia do Desenvolvimento, 21 -23/maio/ 1998, Gramado, RGS, c no $\mathrm{x}^{\wedge} \mathrm{vth}$ Biennial Meetings of the International Society for the Studies of Behavioral Development - ISSBD, Bern, Suiça, 1-4 Julho, 1998.

2 Doutaranda e mestrado no Dpto de Psicologia Experimental/IPUSP, pesquisadora do Centro de Estudos do Crescimento e Desenvolvimento do Ser Humano-CDH FSP-USP. email: neusagso@usp.br

3 Pós-doutoranda e doutorado no Depto de Psicologia Social//PUSP, mestrado em Psicologia Experimental//PUSP, pesquisadora do CDH, FSP-USP e do L^APSI-IPUSP. email: elainepr clas.com.br. Endereço $\mathrm{d}^{\wedge} \mathrm{o}_{\mathrm{o}} \wedge \mathrm{CDH}$ : Av Dr Arnaldo 715 - subsolo - sala 21. CEP 01246-904 São Paulo - s^P Brasil. Tel/fax: 011- 3061-3572. 
Já para REA \& CUKIER (1988), estudando 736 mães que freqüentavam Centros de Saúde, as razões para a introdução de alimentos substitutos ao leite materno foram insuficiência do leite e trabalho fora do lar, concluíndo que o desmame deve ser visto como um processo social multicausado.

Concluindo na mesma direção, SANTOS OLIVEIRA (1996) sugeriu que mães que trabalham fora, cujos filhos permanecem em creches do Estado que dispõem de sala de amamentação, aleitavam pouco (19\% até 4 meses) devido à dupla mensagem dada pela instituição quanto a essa prática.

Vários estudos (BRYANT, 1982; DESANCTIS, 1986; FANUCHI \& FANUCHI, 1985; GROSSMAN ET AL., 1990; WOOLLETT, 1987) concluíram pela importância de fatores socioculturais afetando a decisão da mãe quanto à amamentação, apontando, entre outros, para a importância da rede de apoio, influência de pessoas geograficamente próximas, crenças, aculturação. Para DETTWYLER (1987), a amamentação se revelou um processo variável e complicado, definido pela cultura. A opção para fazer escolhas (ESCRIBA, COLMER, MAS, GRIFOL, 1994) parece ser um fator central na decisão quanto à amamentação, assim como amamentação e trabalho foram apontados como incompatíveis (CHALMERS, RANSOME \& HERMAN, 1990). Em uma outra direção, mas complementando estes esiudos, BOWILLE (1998) apontou para o tipo de apego como influenciando o comportamento alimentar da amamentação associado à presença ou ausência de desnutrição.

Dentro da perspectiva exposta acima de que a amamentação é um processo sócio-cultural multicausado, o presente trabalho objetivou discutir questões atinentes à amamentação comparando algumas situações no Brasil.

Para isso, foram estudados o sistema de amamentação em três contextos de desenvolvimento diverso: um grupo de 60 crianças de famílias de baixa renda, de 0-24 meses de idade, assistidas por um Centro de Saúde, moradoras de uma região urbana da cidade de São Paulo (Vila Madalena: VM); um grupo de 257 crianças paulistanas, de 4-12 meses, freqüentadoras de uma creche no local de trabalho da mãe; um grupo de 28 crianças de 0-3 anos, moradoras da região do Cocal, uma zona rural pobre no interior do Estado nordestino do Piauí (Pi).

Estes três estudos foram realizados independentemente um do outro, dentro de um método eto-etnográfico, observando o comportamento em ambiente naturalístico e descrevendo o ambiente.
O estudo realizado em Vila Madalena foi um estudo longitudinal, de duração de um ano, onde foram aplicados vários instrumentos visando coletar dados sobre o ambiente doméstico, organização familiar e desenvolvimento infantil, tendo como objetivo principal o desenvolvimento de instrumentos para acompanhamento de desenvolvimento em Unidade de Saúde (SIQUEIRA, OLIVEIRA, RABINOVICH \& SANTOS, 1992).

O estudo realizado no Piaui foi um estudo sincrônico, objetivando fornecer subsídios para a ação da Pastoral da Criança, CNBB Brasil, em que foram coletadas informações sobre o desenvolvimento infantil, organização sociofamiliar e ambiente doméstico, assim como videofilmagem e fotos sobre a moradia (RABINOVICH, 1994).

O estudo em creche teve por objetivo estudar a interação mãe-bebê, durante a amamentação, em sala de amamentação em creche, através de observação de videofilmagem das duplas mãebebê, entrevistas com as mães, descrição do ambiente (SANTOS-OLIVEIRA, 1996).

Os dados utilizados no presente trabalho foram obtidos por: entrevista com a mãe; observação e videofilmagem; observação e registro do ambiente; entrevista familiar.

\section{RESULTADOS E DISCUSSÃO}

As crianças foram comparadas quanto a: idade do desmame; motivo do desmame; esquema de amamentação; suplementação à amamentação; locus da amamentação; padronizações normatizadoras.

Quanto à idade do desmame (Tabela 1), enquanto a maioria (72\%) das crianças no Piauí era aleitada após os 3 meses, $44 \%$ em Vila Madalena já estavam desmamadas com esta idade assim como $81 \%$ das crianças ao entrar na creche, aos 4 meses. Na mesma direção, enquanto no Piauí $40 \%$ das crianças continuavam a ser aleitadas após 12 meses, apenas $26 \%$ das crianças de Vila Madalena o eram, e nenhuma na creche.

Tabela 1 - Idade do desmame (\%)

\begin{tabular}{l|c|c|c}
\hline Idade & $0-3$ meses & $3-12$ meses & +12 meses \\
\hline SP & 44 & 30 & 26 \\
Creche & $81(4 \mathrm{~m})$. & 19 & - \\
$\mathrm{Pi}$ & 24 & 43 & 33 \\
\hline
\end{tabular}

O motivo do desmame (Tabela 2) foi atribuído a criança em Vila Madalena (44\%), ou coincidindo com a idade preestabelecida como norma médica, dada pelo Centro de Saúde, de 
desmame aos 6 meses (20\%), ou porque as crianças foram desmamadas logo ao nascer por não aceitarem o peito (12\%). A mãe que decidiu pelo desmame em Vila Madalena (56\%) desmamou aos 3 meses, geralmente por motivo de trabalho.

No Piauí, foi a mãe a decidir pelo desmame, devido à necessidade de trabalhar (37,5\%) e gestação (37,5\%). A gestação como motivo de desmame foi encontrada também no Butão Ocidental (BOHLER \& BERGSTROM, 1995) como a principal razão para o desmame.

$\mathrm{Na}$ creche, o retorno ao trabalho foi a principal motivação para o desmame, sendo que apenas 19\% das crianças das creches eram aleitadas.

Em Vila Madalena, o esquema de amamentação (Tabela 3) preconizado pelo Centro de Saúde era aleitar a pedido, o que foi seguido por $60 \%$ das mães, enquanto as demais $40 \%$ estabelece- ram horário para aleitar. No Piauí, as crianças eram aleitadas a pedido (94\%), acrescido, contudo, de antecipação ao pedido da criança (30\%) e de horário (20\%), de modo a garantir o aleitamento e a relação mãe-criança através dele. Na creche, a amamentação era com horário pré-determinado pela instituição.

No Piauí, desde o nascimento, $87 \%$ das crianças receberam como implementação alimentar o gomoso, um mingau feito de farinha, dado originalmente com o dedo. As crianças que não o receberam foram de mães de maior escolaridade, por influência da norma médica-educacional. Em Vila Madalena, a orientação do Centro de Saúde era de aleitamento exclusivo, o que foi dito ser seguido pelas mães que aleitaram até os 6 meses ou mais. Na creche, todas as crianças receberam suplemento alimentar, segundo orientação do pediatra da instituição.

Tabela 2 - Decisão sobre idade do desmame pela mãe ou criança (\%)

\begin{tabular}{l|c|c|c|c|c|c}
\hline Idade & $0-3$ meses & $0-3$ meses & $3-12$ meses & $3-12$ meses & +12 meses & +12 meses \\
\hline Decisão & criança & mãe & criança & mãe & criança & mãe \\
\hline S. Paulo & 27 & 73 & 70 & 30 & - & - \\
Creche & 0 & 100 & $?$ & $?$ & - & - \\
Piauí & 50 & 50 & 75 & 25 & 0 & 100 \\
\hline
\end{tabular}

Tabela 3 - Esquemas de amamentação: controlado pela mãe ou criança (\%)

\begin{tabular}{l|c|c|c|c|c|c}
\hline Idade & $0-3$ meses & $0-3$ meses & $3-12$ meses & $3-12$ meses & +12 meses & +12 meses \\
\hline Controle & mãe & criança & mãe & criança & mãe & criança \\
\hline S.Paulo & 33,3 & 10,0 & 3,3 & 23,3 & 3,3 & 2,7 \\
Creche & $?$ & $?$ & 100 (creche) & 0 & - & - \\
Piauí & 2,4 & 17,1 & 7,1 & 40,5 & 7,1 & 28,6 \\
\hline
\end{tabular}

Tanto o esquema de amamentação quanto a suplementação alimentar precoce no Piauí podem ser pensadas estar a serviço da sobrevivência tanto da mãe quanto da criança em uma cultura de secas endêmicas e de grande pobreza, conforme já analisado no estudo de SCHEPERHIJGHES (1985).

Quanto ao locas de amamentação, no Piauí, as crianças permaneciam em suas redes ou no colo de quem havia sido designado, na família, para cuidar dela e, face ao choro ou desconforto, era trazida à mãe. Circulava dentro/fora da casa, contatando com quem lá estivesse. A mãe, ao ir trabalhar, deixava a criança em casa, cuidada por um familiar, avó e/ou irmão.

Em Vila Madalena, a criança permanecia no berço ou na sala, e a mãe a atendia segundo o esquema em funcionamento, dentro da casa de poucos cômodos, em meio a seus afazeres domésticos. O trabalho materno externo à casa implicava, em geral, no desmame. À noite, em geral, era oferecida mamadeira, embora as crianças dormissem no quarto dos pais. Embora houvesse uma rede familiar, a vida cotidiana se dava intramuros, havendo uma restrição a contatos exteriores quer do bebê quer da mãe.

Na creche, havia uma sala especial para a amamentação, onde várias mães aleitavam ao mesmo tempo, havendo interações entre as mães e entre os bebês. Não era permitido à mãe entrar na creche quando assim desejasse e, embora a creche fosse localizada em local próximo ao trabalho, não era facilitado que a mãe dele se ausentasse, a não ser nos esquemas predeterminados pela legislação, impedindo, pois, tanto do lado da creche quanto do trabalho, uma adaptação da mãe ao seu bebê.

As padronizações normatizadoras encontradas no Piauí foram normas tradicionais, ancoradas no saber popular e na experiência acumulada, que propugnavam o desmame com a ocorrência c e nova gestação. Esta norma pres- 
supunha também o bebê dormindo ao lado dos pais, de modo que o desmame do peito ocorria antes do afastamento no dormir. A criança só passava a dormir em quarto separado dos pais até ter"entendimento", com 5 anos, sua rede sendo afastada da dos pais com os sucessivos nascimentos.

Esta norma do Piauí coincide com a dos povos da caça e coleta, quando a criança era desmamada com a gravidez, havendo uma diferença de 3, 4 anos entre as gestações, quando ela já poderia locomover-se por si e não depender apenas do leite materno para se nutrir (ANDERSON, 1983). Normas mais recentes no Piauí preconizando aleitamento exclusivo com introdução posterior de sucos e papas, contradiziam estas práticas, sendo fonte de conflitos avós/pais, acompanhadas de introdução do berço. Estas normas, "educando para civilizar", pareciam estar sendo incorporadas, enquanto as tradicionais eram mantidas.

Em Vila Madalena, observou-se vários padrões normatizadores - família, amigas, vizinhas - sendo a norma estabelecida pelo Centro de Saúde de aleitamento exclusivo, até os 6 meses, apenas oficiosamente seguida em vários casos. Parentes e vizinhos, assim como a própria experiência influenciavam na decisão do modo de amamentação, confirmando o estudo de BRYANT (1982) em que o aconselhamento da rede geograficamente próxima afetava o impacto que os profissionais de saúde tinham sobre as decisões maternas sobre as práticas de alimentação.

Tanto o desmame precoce quanto a amamentação tardia emergiram, em Vila Madalena, como fontes de problemas na relação mãe/criança devido, em parte, a conflitos gerados pelo afastamento da norma. A ação normatizadora do Centro de Saúde foi, portanto, uma ação "educadora”, com modelos a serem seguidos, determinando práticas sócio-simbólicas, desvios e sanções, intra e extrasubjetivos. A influência do controle médico negando a validade de respostas mútuas corporais e emocionais dentro da díade mãe criança, apontada por MILLARD (1990), pode ser suposta estar influenciando nos casos de conflitos entre as crenças maternas e as das fontes normatizadoras.

Na creche, a norma foi imposta pela instituição, não possibilitando às mães escolha: ao optar pela creche como solução para a sua permanência no trabalho e uma certa proximidade à criança, são alijadas de outras decisões. Como a opção para fazer escolhas parece ser um fator central na decisão quanto à amamentação (ESCRIBA, COLMER, MAS, GRIFOL, 1994), a amamentação em creche resultaria dificultado.

\section{CONCLUSÃO}

A motivação materna parece ser o principal aspecto a ser considerado no estudo da amamentação. No Piauí, em Vila Madalena e na creche, as mães que amamentaram fizeram-no motivadas e criando situações propiciatórias. A mãe que trabalhava e que optava pela creche onde poderia aleitar exemplifica com clareza este ponto, assim como as mães no Piauí que adotavam vários esquemas de amamentação além de introduzirem um tipo de alimentação que possibilitava à criança ser alimentada em sua ausência. Igualmente, em Vila Madalena, observou-se a retirada da mãe do mercado de trabalho a fim de se dedicar ao neonato, retornando a este com o desmame do filho.

Face à dificuldade de aleitar dado o modo de vida destas mães, é o seu empenho que garante a amamentação. O modo de vida urbano se mostrou especialmente contraditório com a amamentação, em que os valores baseados no individualismo, na privatização da vida e dos espaços, e na busca de autonomia como valor máximo atribuído à subjetividade, ocasionou a separação precoce mãe/criança, consubstanciada na imediata separação pós-parto e na presença de berço em quarto isolado. O valor de autonomia se repete, também, no isolamento ao qual o neonato e infante são deixados, por uma representação do bebê como autosuficiente, em oposição ao visto no Piauí.

A motivação materna, deve-se considerar a presença/ausência de uma rede de apoio à mãe: no Piauí, fornecida pela família extensa; na creche, por ser no local de trabalho da mãe; em Vila Madalena, fornecendo a possibilidade de a mãe poder dispor de seu tempo e energia. A rede de apoio é o aspecto complementar ao empenho materno, de que este depende para se concretizar.

Pode-se observar vários conflitos relacionados às normas, devido a serem elas baseadas em experiência ou conhecimento, por enculturação ou por aculturação, visando educar as mães ou criar os bebês. As mães, frente à autoridade constituída na pessoa do médico ou educador, pareciam seguir normas que realmente não seguiam, mas que criavam conflitos internos e externos, face à dupla mensagem. A dupla mensagem se refere a regras que são impossíveis de serem seguidas e à promoção da amamentação com a ausência de uma realidade concreta onde este pode ocorrer. Face a estas contradições, pode-se observar que a própria experiência parece ser um guia para as mães pois mães de segundos filhos tendem a aleitar por mais tempo e desmamar mais tarde.

Conclui-se, em sse, que a idade de desmame esteve associada à motivação materna e à pre- 
sença/ausência de uma rede de apoio, familiar e social, em que padrões normatizadores são confrontados com a realidade do cotidiano socio-familiar.

O aleitamento materno, portanto, não pode ser visto independentemente do contexto físico e social onde ocorre, que regula os costumes de cuidados, ou seja, as práticas de cuidados. Estas, por sua vez, estão em interação com as representações que se fazem os caretakers sobre o desenvolvimento da criança que, caso entrem em dissonância com o que efetivamente vvem, criam ruídos que acabam por penalizar o próprio desenvolvimento da mesma. É o que ocorre no caso de padronizações normais de dupla mensagem.

O presente estudo sugere que se deve evitar generalizações sobre práticas de amamentação para toda a população sem considerar as variáveis contextuais e que os valores de individualismo, isolamento, autonomia, que separam mãe/criança desde o nascimento, são contraditór Os com o aleitamento materno.

\begin{abstract}
The aim of this study was to compare breast-feeding systems in three different developmental contexts: a group of children aged 0-2 years from low income families living in an urban arca in the city of São Paulo; a group of children aged 4- 12 attending a public day-care centre also in the city of São Paulo; a group of children aged 0-3 years from a poor rural arca in the state of Piauí, Northeast of Brazil. It was an ethos-ethnographic study. Data were obtained through interviews with the mothers about their child and family; observations of mother-child interaction; video recording; registration of the environment. Breast-feeding systems were compared as to: age of weaning; decision about age of weaning; breast-feeding schedule; food supplement; locus of breast-feeding; breast-feeding/weaning standards. It was concluded that: age of weaning was related to mother's motivation and to presence/absence of a supporting network, in which norms were confronted with the sociofamiliar everyday reality; generalisations about breast-feeding practices to the overall population without considering contextual variables should be avoided; western values of individualism, separateness and autonomy seem contradictory in relation to breast-feeding.
\end{abstract}

Key-words: breast-feeding; developmental context; supporting network; maternal motivation; breast-feeding/weaning standards; sociofamiliar everyday reality.

\section{REFERÊNCIAS BIBLIOGRAFICAS}

ANDERSON, P. The reproductive role of the human breast. Current Anthropology, 24(1): 2545, Feb. 1983.

BOHLER, E.; BERGSTROM, S. Premature weaning in East Bhutan: only if mother is pregnant again. Journal of Biosocial Science, 27(3): 253-265, Ju1. 1995.

BOUVILLE, J. F. The influence of family environment on the onset and evolution of child malnutrition in an urban African setting. In: XVth Biennial Meeting of the International Society for the Sudies of Behavioral Development,Berne, 1998. Abstracts, Bern, ISSBD, 1998. p. 44.

BRYANT, C. A. The impact of kin, friend and neighbor networks on infant feeding practices: Cuban, Puerto Rican and Anglo families in Florida. Social Science and Medicina, 16(20): 1757- 1765, 1982.

CHALMERS, B.; RANSOME, O. J.; HERMAN, A. Working while breast feeding among coloured women. Psychological Reports, 67(3 pt 2): 1123-1128, Dec. 1990.

DeSANCTIS, L. Infnt feeding practices of Haitian mothers in South Florida: cultural beliefs and acculturation. Maternal Child Nursing Journal, 15(2): 77-89, Sum.1986.

DETTWYLER, K. A. Breastfeeding and weaning in Mali: cultural context and hard data. Social Science and Medicine, 24(8): 633-644, 1987.

ESCRIBA, V.; COLMER, C.; MAS, R; GRIFOL, $\mathrm{R}$. Working conditions and the decision to breastfeed in Spain. Health Promotion lnternational, 9(4): 251-258,1994.

FANUCHI, L. N.; FANUCHI, N. B. d. A. Mãe rural e alojamento conjunto: avaliação de uma prática no interior. J. Pediatr, 59(5/ó): 483-488, nov.-dez. 1985. [Rio de Janeiro]

GROSSMAN, L. K.; FITZSIN-MONS, S. M.; LARSENALEXANDER, J. B.; SACHS, L. The infant feeding decision in low and upper income women. Clinical Pediatrics, 29(1): 3037, Jan. 1990.

ISLER, H.; LEONE, C.; QUINTAL, V. S. Duração do amamentação materno em uma área urbana de São Paulo, Brasil. Bol. of Sanit. Panam., 106(6): 513-522, 1989.

MILLARD, A. V. The place of clock in pediatric advice: racionares, cultural themes, and impediments to breastfeeding. Social Science and Medicine, 31(2): 211-221, 1990. 
RABINOVICH, E. R O modo de morar e a vida cotidiana como indicadores qualitativos do desenvolvimento infantil: um estudo de uma população de crianças de 0-3 anos na zona rural do Piauí, Brasil. Caderno de Desenvolvimento Infantil, 1(1): p. 47-59, Jul. 1994.

REA, M. E; CUKIER, R Razões de desmame e de introdução da mamadeira: uma abordagem alternativa para seu estudo. Rev. Saúde PúbL, 22(3): 184-191,1988.

SANTOS-OLIVEIRA, N. G. Amamentação em creche no local de trabalho da mãe. São Paulo, 1996. [Dissertação de Mestrado - Departamento de Psicologia Experimental do Instituto de Psicologia da Universidade de São Paulol.
SCHEPER-HUGHES, N. Culture, scarcity, and maternal thinking: maternal detachment and infant survival in a Brazilian shantytown. EhOS, 13(4): 291 -317, Win. 1985.

SIQUEIRA, A. A. E d.; OLIVEIRA, D. C.; RABINVOVICH, E. R; SANTOS, N. G. Instrumentos para o acompanhamento e avaliação do desenvolvimento infantil na Atenção Primária à Saúde. Revista Brasileira de Crescimento e Desenvolvimento Humano, 2(2): 59-99, Jul/Dez. 1992.

VENÂNCIO, S. 1.; MONTEIRO, C. A. A tendência da prática de amamentação no Brasil nas décadas de 70 e 80 . Revista Brasileira Se l ;pidemiologia, 1(1): 40-49,1998.

WOOLLETT, A. Who breastfeeds? The family and cultural context. Journal of Reproductive and Infant Psychology, 5(3): 127- 131, jul-sept. 1987. Especial Issue: Breastfeeding). 